\title{
Plantes alimentaires spontanées de la région du Fromager (Centre- Ouest de la Côte d'Ivoire) : flore, habitats et organes consommés.
}

\author{
N'Dri M.T. KouamÉ ${ }^{*}$, Guy M. Gnahoua ${ }^{2}$, Konan E. KouASsI ${ }^{1} \&$ Dossahoua Traoré $^{1}$ \\ ${ }^{1}$ Laboratoire de Botanique, UFR Biosciences, Université de Cocody, 22 BP. 582 Abidjan 22, Côte d'Ivoire \\ ${ }^{2}$ Centre National de Recherche Agronomique, Station de Recherche de Gagnoa, BP 602 Gagnoa, Côte d'Ivoire. \\ *Auteur pour les correspondances (E-mail : ndrimaritherese@yahoo.fr) \\ Reçu le 14-12-2006, accepté le 08-04-2008.
}

\begin{abstract}
Résumé
Une enquête ethnobotanique, visant à inventorier les plantes spontanées alimentaires encore présentes dans la région du Fromager (Centre-Ouest) de la Côte d'Ivoire, a été menée auprès des populations rurales. Les espèces recensées sont au nombre de 72, réparties entre 61 genres et 42 familles. Les familles les plus représentées sont les Sterculiaceae, les Arecaceae et les Solanaceae. L'habitat de ces plantes spontanées a été déterminé; il s'agit des forêts, des jachères et des vergers de cacaoyers et de caféiers. Les organes les plus consommés sont les fruits (38 \%), les feuilles (33 $\%)$ et les graines $(19 \%)$. Ces enquêtes ont montré qu'il existe encore un nombre élevé d'espèces spontanées alimentaires dans la Région du Fromager.
\end{abstract}

Mots clés: Plantes alimentaires spontanées, habitats, organes consommés, Côte d'Ivoire

\section{Abstract}

Spontaneous food plants of the "Fromager" Region (Western Center of Côte.d'Ivoire): flora, habitats and consumed organs

A ethnobotanic survey, aimed at the inventory of the food spontaneous plants present in the "fromager" region (Western Center) of Côte d'Ivoire, was carried out with the collaboration of the rural populations. The number of species inventoried is 72, belonging to 61 generas and 42 families. The most represented families are Sterculiaceae, Arecaceae and Solanaceae. The habitats of these spontaneous plants species were forests, fallows and the cocoa and coffee plantations. The most consumed organs were fruits (38\%), leaves (33\%) and seeds (19\%). These investigations showed a high number of food spontaneous species in the "fromager" region.

Key words: Spontaneous food plants, habitats, consumed organs, Côte d'Ivoire

\section{Introduction}

En Côte d'Ivoire, environ 12 millions d'hectares de forêt ont disparu, en moins de 40 ans, sous l'effet de l'exploitation forestière anarchique et de l'agriculture itinérante. La couverture forestière, estimée à 15 millions d'hectares en 1960 (Aké Assi \& Boni, 1990), est passée à 2,5 millions d'hectares à la fin des années 1990 et est majoritairement composé de forêts classées et de parcs nationaux (Osseni \& Silué, 1998). Dans le milieu rural, cette déforestation est ressentie à la fois par la diminution des terres cultivables et la raréfaction des plantes alimentaires spontanées. La dégradation intense de la forêt pose le problème de la survie des plantes spontanées alimentaires, véritables sources de compléments nutritionnels et de devises en 
milieu rural. Ces espèces ont joué, par le passé, un rôle important dans la survie des populations, surtout en période de guerre, de sécheresse et d'invasion des cultures par les criquets (GautierBeguin, 1992).

Pour freiner le phénomène de la déforestation, de nombreux efforts ont été consentis pour la sauvegarde des espèces. Malgré ces efforts, l'exploitation forestière se poursuit et écrème les reliques, de toutes les espèces pouvant servir dans le domaine alimentaire. La gestion durable de nombreuses espèces alimentaires spontanées est de plus en plus incertaine. Dans un tel contexte, il est important d'initier des études ethnobotaniques, en vue d'aboutir à la connaissance, à la conservation et à la valorisation de ces espèces.

En ethnobotanique, plusieurs travaux sur les plantes alimentaires spontanées ont fait l'objet de nombreuses études en Afrique (Falconer, 1990 ; Malaise, 1997). En Côte d'Ivoire, les recherches de N'Dri (1986), Gautier-Beguin (1992), N'guessan (1995), Kouamé et Ambé (2001) (2000) ont été réalisés chez les Dida, les Baoulé, les Krobou, les Senoufo et chez les Gagou et les Gouro.

Chez les Bété, aucune étude de ce genre n'a encore été menée et c'est ce qui justifie le choix de la Région du Fromager (Centre-Ouest) en Côte d'Ivoire, pour cette étude.

Le présent travail, réalisé dans le Département de Gagnoa, est une contribution aux précédentes études ethnobotaniques. Ce travail vise à recenser les plantes alimentaires spontanées de la zone forestière de la Région du Fromager. Dans ce recensement, nous avons pris soin de noter les organes consommés et les biotopes qui abritent les plantes

\section{Site d'étude}

La présente étude a été conduite dans la Région du Fromager, précisément dans le Département de Gagnoa, au Centre-Ouest de la Côte d'Ivoire. La Région du Fromager comprend deux Départements : Gagnoa (Chef lieu de Région) et Oumé. Le Département de Gagnoa est localisé dans le bassin versant du fleuve Sassandra, avec une superficie de $2500 \mathrm{~km}^{2}$ (Anonyme, 2001). II est compris entre $5^{\circ} 40$ et $6^{\circ} 10$ de latitude Nord et entre $5^{\circ} 50$ et $6^{\circ} 20$ de la longitude Ouest (Andriesse et al., 1994). Le Département de Gagnoa comprend cinq sous-préfectures : Gagnoa, Bayota, Ouragahio, Guibéroua et Gnagbodougnoa (Figure 1). Entre 1994 et 2004, la température moyenne annuelle a été de $27^{\circ} \mathrm{C}$ avec une pluviométrie moyenne annuelle de 1402 mm (Koné, 2004). Les formations pédologiques du Département de Gagnoa sont dominées par des sols ferrallitiques fortement désaturés (Monnier, 1983). La végétation originelle était une forêt dense humide semidécidue (Guillaumet \& Adjanohoun, 1971). A la place de cette végétation, se dresse aujourd'hui une mosaïque de lambeaux forestiers, des jachères à Chromolaena odorata, et des plantations (Gnahoua, 2004).

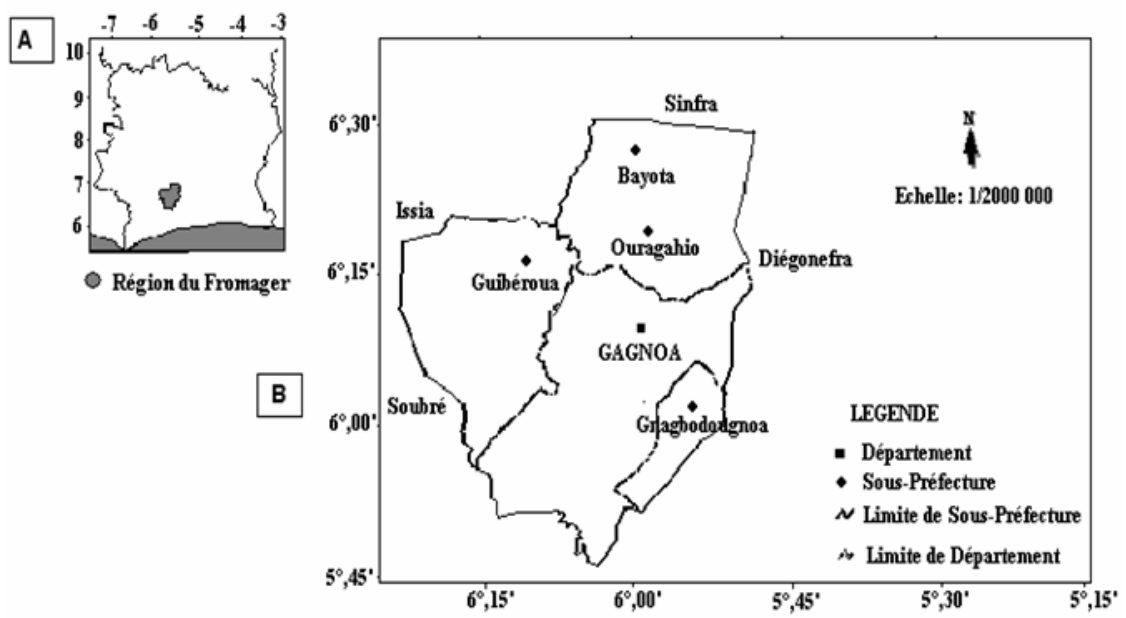

Figure 1 : Carte de la situation géographique et administrative de la zone d'étude (Source : BNETD/OCT)

A : Situation de la région du Fromager par rapport à la Côte d'Ivoire

$B$ : Situation du Département de Gagnoa dans la Région du Fromager 


\section{Matériels et méthodes}

\subsection{Matériel biologique}

Le matériel biologique est constitué de plantes alimentaires spontanées (arbres, arbustes, lianes et herbes) dont les organes sont consommés. Ces organes sont: les feuilles ou les bourgeons, les fruits, les graines ou les amandes, les écorces, les pédoncules, les tubercules, les tiges, etc.

\subsection{Méthode d'étude}

Avant les enquêtes ethnobotaniques proprement dites, des prospections ont été effectuées dans le Département en vue du choix des souspréfectures et des villages. Trois souspréfectures ont été choisies en fonction de leurs positions géographiques par rapport à la ville de Gagnoa. Ce sont Gnagbodougnoa (Sud-est), Ouragahio (Nord) et Guibéroua (Ouest). Dans chaque sous-préfecture, trois villages ont été retenus par rapport à la distance les séparant $(10$ à $30 \mathrm{Km})$. L'enquête s'est basée sur l'interview de 12 personnes par village, choisies au hasard, soit 108 personnes au total. Nous avons soumis une liste de questionnaires aux personnes interrogées. Ces questionnaires devaient nous permettrent de savoir quelles sont les espèces consommées qui sont récoltées en brousse? Quelles sont les parties consommées? Où pouvons-nous les trouvées ; dans les jachères, dans les forêts ou dans les plantations? II était aussi nécessaire de savoir si ces espèces étaient disponibles en quantité.

Sur le terrain, il est courant qu'un même nom soit attribué à plusieurs espèces ou l'inverse. Ainsi, le recensement des plantes alimentaires spontanées à partir des noms locaux seulement comporte des risques d'erreurs. Ces risques sont minimisés, par la récolte d'échantillon d'organes consommés et de parties aériennes pour la confection d'un herbier. Lorsque cela était possible, des photographies ont été faites sur les sites de récoltes ou sur les marchés locaux pour une meilleure identification de ces espèces. Les espèces inventoriées ont été reparties en fonction des milieux écologique (forêt, jachère, plantation et ubiquiste).

\subsection{Méthode d'analyse}

\subsubsection{Espèces inventoriées}

Nous avons dressé une flore des plantes alimentaires spontanées du Département de Gagnoa, en prenant en compte toutes les espèces inventoriées sur le terrain. Pour nommer les taxons, la flore de Hutchinson et Dalziel (19541972) a été utilisée. Les ouvrages de Lebrun et Stock $(1991,1992,1995,1997)$ et ceux de Aké Assi $(2001,2002)$ ont servi à actualiser les taxons.

\subsubsection{Niveau de connaissance et d'exploitation des espèces alimentaires spontanées}

Afin de mieux présenter les résultats obtenus, nous avons combiné les critères de connaissance et de consommation effective selon Ambé (2001). Le niveau de la connaissance villageoise relative (Cr. \%) pour chaque espèce a été estimé par le rapport entre le nombre de personnes connaissant l'espèce $(n)$ et le nombre total de personnes interrogées $(\mathrm{N})$. Elle est traduite par la formule suivante :

$\mathrm{Cr}=(\mathrm{n} / \mathrm{N}) \times 100$

La méthode de Dajoz (1982) a permis de répartir les espèces en trois groupes : le premier groupe, de 50 à $100 \%$, comprend les espèces les plus connues; le deuxième groupe, de 25 à $50 \%$, renferme les espèces moyennement connues, et le troisième groupe, de 0 à $25 \%$, compte les espèces peu connues.

\section{Résultats}

\subsection{Espèces inventoriées}

Les plantes spontanées alimentaires inventoriées sont au nombre de 72 espèces (Annexe 2). Elles se répartissent entre 61 genres et 42 familles. La sous-préfecture de Gnagbodougnoa totalise 59 espèces, celle de Guibéroua 48 et 44 pour celle de Ouragahio. Les familles les plus représentées sont les Sterculiceae (5 espèces), les Arecaceae et les Solanaceae (4 espèces chacune).

Les espèces inventoriées sont représentées par $39 \%$ d'arbres, les arbustes comptent $17 \%$, les herbes $19 \%$ et les lianes ne représentent que 24 $\%$. Par niveau de connaissance et d'exploitation, les espèces alimentaires spontanées identifiées sont divisées en trois groupes. 


\subsection{Les espèces les plus connues}

Les espèces plus connues sont au nombre de 7 et leurs pourcentages varient entre 64,81 à $100 \%$. Ce sont : Irvingia gabonenis, Myrianthus arboreus, Myrianthus libericus, Myrianthus serratus, Ricinodendron heudelotii, Beilschmiedia mannii et Zanthoxylum gilletii (Tableau 1). Ces espèces ont été consommées au moins une fois par les personnes interviewées. Les caractéristiques communes à ces plantes semblent être le goût (généralement très apprécié) et leur disponibilité toute l'année ou au moins durant une grande partie de l'année. Parmi les espèces bien connues, les graines de Irvingia gabonenis, Ricinodendron heudelotii et Beilschmiedia mannii sont les plus recherchés. Ces graines se conservent plus facilement une fois séchées. Elles sont disponibles presque toute l'année sur les marchés locaux. Les jeunes feuilles de Myrianthus arboreus, Myrianthus libericus, Myrianthus serratus et Zanthoxylum gilletii sont consommées rapidement une fois récoltées. Ces jeunes feuilles fraîches sont difficiles à conserver si bien que la commercialisation reste limitée.

\subsection{Les espèces moyennement connues}

Elles sont au nombre de 13 et leur niveau de connaissance est compris entre 25 et $47 \%$. Ce sont : Cola millenii, Cola gigantea, Cola lateritia, Treculia africana, Strombosia pustulata, Dacryodes klaineana, Wissadula amplissima, Solanum nigrum, Raphia hookeri, Laccosperma secundiflorum, Calamus deërratus, Spondias mombin et Grewia mollis (Tableau 1). Parmi ces espèces, 4 ont leurs organes vendus sur les marchés locaux. Ce sont: Strombosia pustulata, Dacryodes klaineana, Solanum nigrum et Treculia africana. Cependant 9 (Cola gigantea, Cola lateritia, Cola millenii, Spondias mombin, Wissadula amplissima, Grewia mollis, Raphia hookeri, Laccosperma secundiflorum et Calamus deërratus) ont leurs organes qui font l'objet de consommation locale et saisonnière. La consommation de Raphia hookeri est plus répandue dans la sous-préfecture de Guibéroua qu'à Ouragahio et à Gnagbodougnoa (Tableau 1). Quant aux organes de Laccosperma secundiflorum et de Calamus deërratus, ils sont plus consommés à Guibéroua et à Gnagbodougnoa.

\subsection{Les espèces peu connues}

Cinquante trois (52) espèces consignées dans le Tableau 1 sont peu connues pour la consommation de leurs organes. Certaines espèces se révèlent comme très peu connues par la population locale, mais exploitées et même vendues sur les marchés locaux. II s'agit de Corchorus olitorus, Piper guineense, Xylopia aethiopica, Garcinia kola, Solanum indicum, Coula edulis, Landolphia hirsuta et Landolphia owariensis. Les fruits de Piper guineense et de Xylopia aethiopica sont consommés sous forme d'épices dans les grillades et les sauces à base de tomates. Les graines de Garcinia kola sont consommées souvent par les hommes adultes à cause de leur vertu afrodisiaque. Certaines espèces sont devenues rares dans la végétation de la Région du Fromager, c'est le cas de Coula edulis, Landolphia hirsuta, Landolphia owariens, Trichoscypha arborea et Telfairia occidentalis.

\subsection{Habitats des espèces}

Les différents milieux écologiques abritant les espèces inventoriées sont présentés dans le Tableau 1. En forêt, 17 espèces ont été recensées, soit $23,28 \%$ des taxons. Les espèces ubiquistes représentent $36 \%$ et les espèces de jachères $19,18 \%$. Quinze pour cent (15\%) des espèces se rencontrent à la fois en forêt et dans les jachères. Dans les plantations (Caféiers et Cacaoyers) et dans les jachères, $4 \%$ des espèces ont été recensés. Les espèces plus connues, Irvingia gabonenis, Myrianthus arboreus, Myrianthus libericus, Myrianthus serratus, Ricinodendron heudelotii et Beilschmiedia mannii, se rencontrent dans tous les milieux (ubiquistes) sauf Zanthoxylum gilletii qui a été identifiée en forêt et en jachère. 
Tableau 1: répartition des espèces recensées par fréquence de reconnaissance par sous-préfecture

\begin{tabular}{|c|c|c|c|c|c|c|c|c|}
\hline $\mathbf{N}^{\circ}$ & Espèces & $A$ & $\mathrm{~B}$ & $\mathrm{C}$ & $\begin{array}{c}\% \text { de } \\
\text { présence }\end{array}$ & Organes consommés & $\begin{array}{l}\text { Habitatts } \\
\text { des } \\
\text { espèces }\end{array}$ & \\
\hline 1 & Irvingia gabonensis & 36 & 36 & 36 & 100,00 & Amandes & $U$ & \multirow{7}{*}{$\begin{array}{l}\text { Espèces } \\
\text { bien connues }\end{array}$} \\
\hline 2 & Myrianthus arboreus & 30 & 33 & 26 & 82,41 & Bourgeons, Fruits & $U$ & \\
\hline 3 & Myrianthus libericus & 30 & 33 & 26 & 82,41 & Jeunes feuilles & $U$ & \\
\hline 4 & Myrianthus serratus & 30 & 33 & 26 & 82,41 & Jeunes feuilles & $U$ & \\
\hline 5 & Ricinodendron heudelotii & 33 & 27 & 29 & 82,41 & Graines & $U$ & \\
\hline 6 & Beilschmiedia mannii & 26 & 32 & 21 & 73,15 & Fruits & $U$ & \\
\hline 7 & Zanthoxylum gilletii & 29 & 26 & 15 & 64,81 & Jeunes feuilles & $\mathrm{F}, \mathrm{J}$ & \\
\hline 8 & Cola millenii & 12 & 25 & 14 & 47,22 & Fruits, jeunes feuilles & $\mathrm{F}, \mathrm{J}$ & \multirow{8}{*}{$\begin{array}{l}\text { Espèces } \\
\text { moyennement } \\
\text { connues }\end{array}$} \\
\hline 9 & Cola gigantea & 12 & 25 & 14 & 47,22 & Arilles, jeunes feuilles & $\mathrm{F}, \mathrm{J}$ & \\
\hline 10 & Cola lateritia & 12 & 25 & 14 & 47,22 & Fruits & $\mathrm{F}, \mathrm{J}$ & \\
\hline 11 & Treculia africana & 17 & 18 & 15 & 46,30 & Fruits & $U$ & \\
\hline 12 & Strombosia pustulata & 21 & 12 & 13 & 42,59 & Graines & $\mathrm{F}, \mathrm{P}$ & \\
\hline 13 & Dacryodes klaineana & 12 & 13 & 19 & 40,74 & Fruits, Bourgeons & $U$ & \\
\hline 14 & Wissadula amplissima & 17 & 21 & 2 & 37,04 & Ecorses & $\mathrm{F}$ & \\
\hline $\begin{array}{l}15 \\
16 \\
17 \\
18 \\
19 \\
20 \\
\end{array}$ & $\begin{array}{l}\text { Solanum nigrum } \\
\text { Raphia hookeri } \\
\text { Laccosperma secundiflorum } \\
\text { Calamus deerratus } \\
\text { Spondias mombin } \\
\text { Grewia mollis } \\
\end{array}$ & $\begin{array}{c}10 \\
9 \\
5 \\
5 \\
13 \\
0 \\
\end{array}$ & $\begin{array}{l}14 \\
23 \\
17 \\
17 \\
12 \\
17 \\
\end{array}$ & $\begin{array}{c}13 \\
4 \\
12 \\
12 \\
7 \\
14 \\
\end{array}$ & $\begin{array}{l}34,26 \\
33,33 \\
31,48 \\
31,48 \\
29,63 \\
28,70 \\
\end{array}$ & $\begin{array}{l}\text { Feuilles } \\
\text { Fruits, Bourgeons, Sèves } \\
\text { Bourgeon terminal } \\
\text { Bourgeon terminal } \\
\text { Fruits } \\
\text { Ecorses } \\
\end{array}$ & $\begin{array}{l}J \\
U \\
F \\
F \\
U \\
J\end{array}$ & \\
\hline 21 & Dioscorea odoratissima & 7 & 9 & 11 & 25,00 & Tubercules & $\mathrm{F}$ & \multirow{23}{*}{$\begin{array}{l}\text { Espèces peu } \\
\text { connues }\end{array}$} \\
\hline 22 & Zanthoxylum rubescens & 0 & 18 & 4 & 20,37 & Jeunes feuilles & $\mathrm{F}, \mathrm{J}$ & \\
\hline 23 & Bombax buonopozense & 0 & 12 & 5 & 15,74 & Pédoncules & $\mathrm{F}, \mathrm{J}$ & \\
\hline 24 & Momordica cabraeï & 12 & 4 & 1 & 15,74 & Graines, Feuilles & $\mathrm{F}, \mathrm{J}$ & \\
\hline 25 & Salacia oliveriana & 11 & 0 & 6 & 15,74 & Feuilles, Graines & $\mathrm{U}$ & \\
\hline 26 & Chrysophyllum perpulchrum & 10 & 4 & 1 & 13,89 & Fruits & $U$ & \\
\hline 27 & Corchorus olitorus & 3 & 8 & 4 & 13,89 & Feuilles, Fruits & $J$ & \\
\hline 28 & Coula edulis & 0 & 8 & 7 & 13,89 & Amandes & $U$ & \\
\hline 29 & Deinbollia pinnata & 4 & 8 & 2 & 12,96 & Fruits & $J$ & \\
\hline 30 & Sesamum indicum & 1 & 7 & 6 & 12,96 & Graines & $J$ & \\
\hline 31 & Triplochiton scleroxylon & 0 & 7 & 7 & 12,96 & Jeunes feuilles & $U$ & \\
\hline 32 & Piper guineense & 3 & 4 & 6 & 12,04 & Fruits & $\mathrm{F}$ & \\
\hline 33 & Ceiba pentandra. & 1 & 5 & 5 & 10,19 & Jeunes feuilles & $\mathrm{U}$ & \\
\hline 34 & Landolphia hirsuta & 6 & 0 & 3 & 8,33 & Fruits & $\mathrm{F}$ & \\
\hline 35 & Parkia bicolor & 1 & 0 & 8 & 8,33 & Graines, Pulpe & $\mathrm{F}, \mathrm{J}$ & \\
\hline 36 & Calpocalyx brevibracteatus & 0 & 0 & 8 & 7,41 & Graines & $\mathrm{F}, \mathrm{J}$ & \\
\hline 37 & Sterculia tragacantha & 1 & 5 & 2 & 7,41 & Amandes, jeunes feuilles & $U$ & \\
\hline 38 & Capsicum frutescens & 4 & 0 & 3 & 6,48 & Fruits & $J, P$ & \\
\hline 39 & Elaeis guineensis & 1 & 3 & 3 & 6,48 & Fruits, Bourgeons, Sève & $U$ & \\
\hline 40 & Xylopia aethiopica & 0 & 1 & 6 & 6,48 & Fruits & $U$ & \\
\hline 41 & Calpocalyx aubrevillei & 0 & 3 & 3 & 5,56 & Graines & $\mathrm{F}, \mathrm{J}$ & \\
\hline 42 & Trichoscypha arborea & 0 & 1 & 5 & 5,56 & Fruits & $\mathrm{F}$ & \\
\hline 43 & Discorea praehensilis. & 1 & 3 & 1 & 4,63 & Tubercules & $U$ & \\
\hline
\end{tabular}


Suite du tableau

\begin{tabular}{|c|c|c|c|c|c|c|c|c|}
\hline $\mathbf{N}^{\circ}$ & Espèces & $A$ & $\mathrm{~B}$ & C & $\begin{array}{c}\% \text { de } \\
\text { présence }\end{array}$ & Organes consommés & $\begin{array}{l}\text { Habitatts } \\
\text { des } \\
\text { espèces }\end{array}$ & \\
\hline 44 & Landolphia owariensis & 2 & 0 & 3 & 4,63 & Fruits & $\mathrm{F}$ & \multirow{29}{*}{$\begin{array}{l}\text { Espèces } \\
\text { peu } \\
\text { connues }\end{array}$} \\
\hline 45 & Acalypha ciliata & 4 & 0 & 0 & 3,70 & Feuilles & $U$ & \\
\hline 46 & Blighia sapida & 0 & 1 & 3 & 3,70 & Arilles & $U$ & \\
\hline 47 & Buchholzia coriacea & 4 & 0 & 0 & 3,70 & Noix & $\mathrm{F}$ & \\
\hline 48 & Carpolobia lutea & 0 & 0 & 4 & 3,70 & Fruits & $\mathrm{F}$ & \\
\hline 49 & Tieghemella heckelii & 0 & 4 & 0 & 3,70 & Amandes & $\mathrm{F}$ & \\
\hline 50 & Garcinia kola & 3 & 0 & 0 & 2,78 & Grainess & $U$ & \\
\hline 51 & Glyphaea brevis & 0 & 3 & 0 & 2,78 & Fruits & $\mathrm{J}$ & \\
\hline 52 & Ipomea obscura & 0 & 0 & 3 & 2,78 & Fruits & $J$ & \\
\hline 53 & Telfairia occidentalis & 0 & 2 & 1 & 2,78 & Graines & $\mathrm{F}$ & \\
\hline 54 & Tetracera alnifolia & 3 & 0 & 0 & 2,78 & Sève & $\mathrm{F}$ & \\
\hline 55 & Thaumatococcus daniellii & 1 & 2 & 0 & 2,78 & Fruits & $\mathrm{F}, \mathrm{J}$ & \\
\hline 56 & Aframomum septrum & 0 & 0 & 2 & 1,85 & Fruits & $\mathrm{F}$ & \\
\hline 57 & Cayratia gracilis & 0 & 0 & 2 & 1,85 & Fruits & $\mathrm{J}$ & \\
\hline 58 & Cissus adenocaulis & 0 & 0 & 2 & 1,85 & Graines & $\mathrm{U}$ & \\
\hline 59 & Dioscorea smilacifolia & 0 & 0 & 2 & 1,85 & Tubercules & $U$ & \\
\hline 60 & Ocimum gratissimum & 0 & 0 & 2 & 1,85 & Feuilles & $\mathrm{J}$ & \\
\hline 61 & Solanum indicum & 0 & 2 & 0 & 1,85 & Fruits & $\mathrm{J}$ & \\
\hline 62 & Solanum torvum & 2 & 0 & 0 & 1,85 & Fruits & $\mathrm{J}$ & \\
\hline 63 & Sphenocentrum jollyanum & 0 & 0 & 2 & 1,85 & Fruits & $\mathrm{F}$ & \\
\hline 64 & Bidens pilosa & 0 & 1 & 0 & 0,93 & Feuilles & $J$ & \\
\hline 65 & Cleome ciliata & 0 & 0 & 1 & 0,93 & Feuilles & $J$ & \\
\hline 66 & Clerodendrum splendens & 0 & 0 & 1 & 0,93 & Feuilles & $J, P$ & \\
\hline 67 & Cucumeropsis mannii & 0 & 0 & 1 & 0,93 & Fruits, jeunes feuilles & $\mathrm{J}, \mathrm{P}$ & \\
\hline 68 & Isolona campanulata & 1 & 0 & 0 & 0,93 & Feuilles & $\mathrm{F}$ & \\
\hline 69 & Justicia flora & 0 & 0 & 1 & 0,93 & Feuilles & $U$ & \\
\hline 70 & Laportea aeatuans & 0 & 0 & 1 & 0,93 & Fleurs & $U$ & \\
\hline 71 & Passiflora foetida & 1 & 0 & 0 & 0,93 & Fruits & $J$ & \\
\hline 72 & Psychotria psychotrioides & 1 & 0 & 0 & 0,93 & Feuilles & $\mathrm{F}, \mathrm{P}$ & \\
\hline
\end{tabular}

NB : $A$ : Ouragahio, $B$ : Guibéroua, $C$ : Gnagbodougnoa, $\boldsymbol{F}:$ forêt ; $\boldsymbol{J}$ : jachère ; $\boldsymbol{P}:$ plantation ; $\boldsymbol{U}:$ forêt, jachère et plantation

\subsection{Organes consommés}

D'une manière générale, les fruits sont les organes les plus consommés (Figure 1). Ils représentent $37,50 \%$ des espèces rencontrées. Les feuilles ou bourgeons (Figure 2) et les graines (Figure 3 ) représentent respectivement
32,88 et $19,18 \%$. Quant aux autres organes (écorces, tubercules, tiges, etc.), ils proviennent de $20,55 \%$ des espèces recensées. Mais les organes qui rentrent fréquemment dans la préparation des repas familiaux sont les feuilles. Les personnes interrogées disent les consommer au moins 2 à 3 fois dans la semaines. 


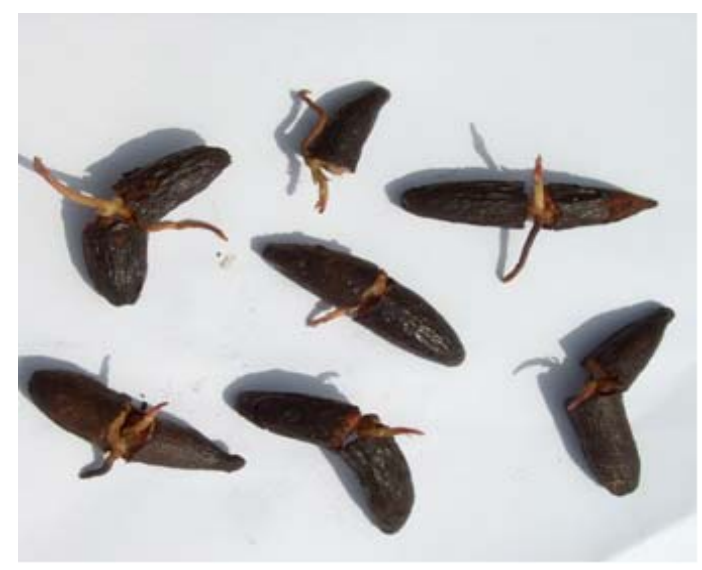

Figure 1: Fruits germés de Beilschmiedia mannii (Meisn) Benth. et Hook ; (Lauraceae)

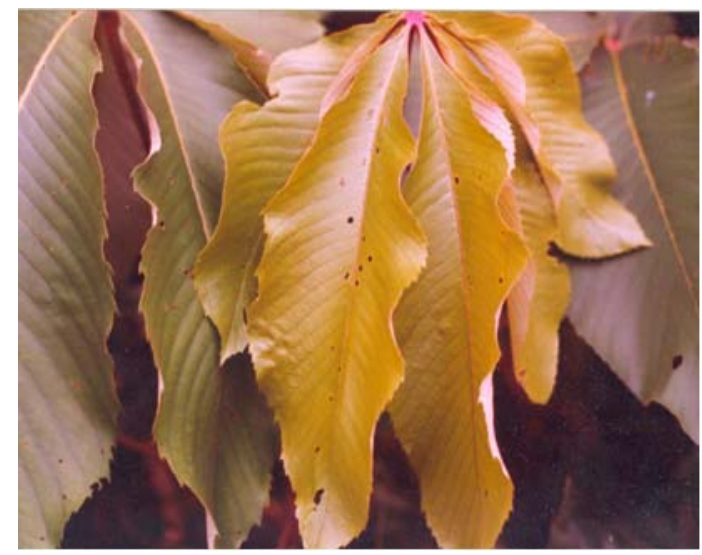

Figure 2: Jeunes feuilles de Myrianthus arboreus $P$. Beauv. (Euphorbiaceae)

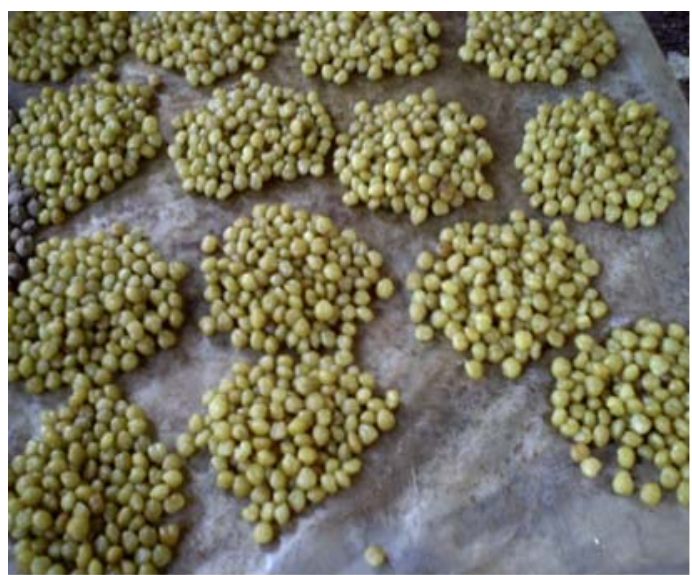

Figure 3: Graines de Ricinodendron heudelotii (Baill.) Pierre ex Pax (Euphorbiaceae)

\section{Discussion}

Du point de vue floristique, il y a eu plus de plantes alimentaires spontanées recensées dans la sous-préfecture de Gnagbodougnoa que Guibéroua et Ouragahio. Les faibles effectifs d'espèces recensées dans ces deux dernières sous-préfectures s'expliquent par la non consommation de certaines espèces citées à Gnagbodougnoa. Par exemple: Aframomum sceptrum et Carpolobia lutea ont été citées parmis les espèces alimentaires spontanées de Gnagbodougnoa alors qu'elles ne sont pas consommées à Guibéroua et à Ouragahio. La dégradation de la végétation peut être aussi une des causes du faible nombre de plantes alimentaires spontanées dans ces deux souspréfectures.

Les espèces plus connues de la flore spontanée du Département de Gagnoa sont surtout présentent dans les forêts, les jachères et dans les plantations. Elles ne se retrouvent en plantation que quand elles sont épargnées par les paysans. Irvingia gabonensis, Ricinodendron heudelotii et Beilschmedia mannii sont très connues car elles produisent des graines que l'on peut conserver et commercialiser toute l'année. Elles sont signalées par N'Dri (1986) à Divo, N'guessan (1995) chez les Krobou et Kouamé (2000) dans le département de Oumé comme étant les principales espèces alimentaires. Ces arbres produisent des amandes très recherchées pour la préparation des sauces. Leur récolte donne lieu à une importante activité commerciale qui occupe les femmes rurales de ces régions (Tabuna, 1999). Pour le reste des espèces à niveau de connaissance élevé, ce sont les bourgeons ou les feuilles qui sont recherchés. La présence de ces organes tout au long de l'année fait d'eux, des produits de grande consommation. Dans cette catégorie d'espèces bien connues, certaines sont protégées, entretenues et mêmes plantées par quelques agriculteurs. Cette attitude montre le niveau d'importance et d'intérêt accordés à ces plantes et le souci d'éviter leur extinction. Pour la catégorie comprise entre 0 et $25 \%$, les espèces alimentaires spontanées peuvent être considérées comme des espèces peu connues. La plupart de ces espèces ont des organes qui sont récoltés pour une consommation individuelle en collation et/ou pendant les périodes de disette. Ces espèces sont très rarement 
commercialisées. Cela a été signalé par N'dri (1986), Gautier-beguin (1992) et Malaise (1997).

Les espèces alimentaires spontanées rencontrées poussent d'elles même et n'ont pas besoin d'entretien particulier. Cette situation fait que leur nombre est plus important dans les forêts et dans les jachères que dans les plantations. Pour ces raisons, l'action de défrichement de la forêt et l'entretien des cultures sont des menaces pour la survie de ces plantes. Busson (1965) en Afrique Occidentale et Noumi (1984) en Afrique Centrale ont fait les mêmes constats.

Dans les plantations de caféiers et de cacaoyers, les espèces alimentaires arborées sont les plus rencontrés. Ce sont: Irvingia gabonensis, Beilschmedia mannii, Myrianthus arboreus, Zanthoxylum gilletii, Strombosia pustulata, Spondias mombin, Garcinia kola, Ricinodendron heudelotii, Treculia africana, etc. Ces observations sont proches de celles de Gautier-Beguin (1992) et de Kouamé (2000). Ces espèces jouent un rôle important dans les formations forestières secondaires et peuvent donner lieu à des systèmes d'agroforesterie.

Dans le Département de Gagnoa, les fruits sont de façon générale, les organes les plus consommés. Ils représentent $37,50 \%$ des espèces rencontrées. Gautier-Beguin (1992), Tra $\mathrm{Bi}$ (1997) et Ouattara (1999) ont déjà signalé cet état de fait. Selon ces auteurs, il existe des variations considérables dans l'abondance des fruits et leurs consommations d'une zone écologique à l'autre. La forte consommation des fruits est due à leur mésocarpe juteux. Pour eux ce sont les enfants qui, en général, en consomment le plus.

Dans la confection des repas familiaux, les feuilles sont plus recherchées. Elles représentent environ $41,18 \%$ des organes fréquemment consommés. Les feuilles sont les organes permanents sur les plantes et donc disponibles sur toute l'année. Le plus souvent, elles servent de base aux soupes, aux ragoûts et servent également de condiments.

\section{Conclusion}

Les enquêtes conduites dans trois souspréfectures de la Région du Fromager, Gagnoa, ont parmi de dresser la liste des espèces spontanées alimentaires. Celles-ci ont été observées dans les milieux écologiques divers: forêts, jachères, plantations pérennes. Les formes biologiques qui produisent les organes couramment consommés sont les arbres. Les espèces les plus connues (50 à $100 \%$ ) sont au nombre de sept (7). Trois milieux écologiques abritent les 72 espèces alimentaires spontanées recensées. Les fruits sont les organes les plus consommés, mais dans l'ensemble, les organes qui rentrent dans la préparation de mets familiaux sont les feuilles. Les graines sont des organes moins utilisées dans les mets $(17 \%$.). Les fruits et les autres organes (30\% environ) font l'objet d'une consommation d'importance intermédiaire.

Au cours des enquêtes, nous avons constaté que certaines espèces comme Solanum nigrum, Ricinodendron heudelotii, Irvingia gabonensis, Beilschmedia manii, Myrianthus arboreus font l'objet d'une commercialisation importante. L'exploitation de ces espèces est en grande partie une activité menée par les femmes, elle concourt à réduire la pauvreté en milieu rural. Car il serait important de sensibiliser les villageois à la gestion de ces ressources naturelles pour contribuer à l'amélioration de leur condition de vie.

\section{Références citées}

Aké Assi L., 2001. Flore de la Côte d'lvoire 1, catalogue, systématique, biogéographie et écologie. Conservatoire et Jardin Botanique, Genève, Switzerland, Boissiera 57, 396 pp.

Aké Assi L., 2002. Flore de la Côte d'lvoire 2, catalogue, systématique, biogéographie et écologie. Conservatoire et Jardin Botanique, Genève, Switzerland, Boissiera 58, 401 pp.

Aké Assi L. \& Boni D., 1990. Développement agricole et protection de la forêt : quel avenir pour la forêt ivoirienne ? Compte rendu de la XIleme réunion plénière de l'AETFAT symposium II: pp 169-176.

Ambé G.A., 2001. Les fruits sauvages comestibles des savanes guinéennes de Côte d'lvoire : état de la connaissance par une population locale, les Malinké. Biotechnol. Agron. Soc. Environ. 5 (1) : 43-58.

Andriesse W., Fresco L.O., Van Duivenbooden N., Windmeijer P.N., 1994. Multi- scale characterization of inland valley agro-ecosystems in West Africa. Netherlands J. Agric. Sci, 42 (2): 159-179. 
Anonyme, 2001. Rapport d'activités de MINAGRA 2000-2001, Gagnoa, Cote d'Ivoire, 20 pp

Busson F., 1965. Plantes alimentaires de l'Ouest Africain. Etude botanique, biologique et chimique. Ministère de la Recherche Scientifique et des Armées, Paris, 568 pp.

Dabbadie M. L., 1996. Etude de la viabilité d'un pisciculture rurale à faible niveau d'Intrant dans le Centre-Ouest de la Côte d'Ivoire: approche du réseau tropicale. Thèse de Doctorat (spécialité océanologique biologique) Université de Paris 6, $207 \mathrm{pp}$.

Dajoz, 1982. Précis d'écologie. Ecologie fondamentale et appliquée Ed. Gauthier-Villers paris $503 \mathrm{pp}$

Falconer J., 1990. The major significance of "minor" forest products. Community forestry note 6 , FAO Rome, $232 \mathrm{pp}$.

Gautier-Beguin D, 1992. Etude ethnobotanique des plantes de cueillettes à utilisation alimentaire dans un village au Sud du V-Baoulé (Côte d'Ivoire centrale). Thèse de Doctorat ès Sciences Techniques, mention biologique. Université de Genève, 368 pp.

Gnahoua G. M., 2004. Contribution des Légumineuses à la régénération des jachères: Intérêts et limites des arbres fixateurs d'azote en zone forestière de la Côte d'Ivoire. Thèse de Doctorat d'Ingénieur, option : Agronomie, UFR STRM, Université Nationale de Côte d'Ivoire, Abidjan, 143 pp.

Guillaumet J. L. \& Adjanohoun E., 1971. La végétation. In le milieu naturel de la Côte-d'Ivoire Mémoire n`50 Paris : ORSTOM, pp 161-262.

Hutchinson J. \& Dalziel J.M. (1954-1972). Flora of West Tropical Africa. Volume I part 1, I part 2, II, III part 1, III part 2, Edition Crown Agents, Londres, Royaume Uni. 295 p., 828 p., 544 p., 276 p., 574 p.

Koné D., 2004. Situation agroclimatique de la station CNRA/Gagnoa de 1971 à 2003. Note Technique, $68 \mathrm{pp}$.

Kouamé N. M. T., 2000. Contribution à l'étude des plantes spontanées alimentaires du department d'Oumé (Côte d'Ivoire). Mémoire de D.E.A d'Ecologie tropicale (Option : Végétale). Université de Cocody Abidjan, Côte d'Ivoire, 122 pp.

Lebrun J. P., Stork A. L., (1991, 1992, 1995, 1997). Enumération des plantes à fleurs d'Afrique tropicale. Genève Conservatoire et Jardin Botanique de Genève, 1-4.

Malaise F., 1997. Se nourrir en forêt claire Africaine. Approche écologique et nutritionnelle. Gembloux, Belgique : Presses agronomiques de Gembloux; Wageningen, Pays-Bas : CTA, 384 pp.

Monnier Y., 1983. Carte de la végétation de la Côte d'Ivoire. In : Vennetier P. Laclavere G. (eds.). Atlas de la Côte d'Ivoire. $2^{\mathrm{e}}$ éd., Jeune Afrique, Paris, France, $72 \mathrm{p}$.

N'Dri P., 1986. Contribution à l'étude de quelques plantes alimentaires spontanées de la région de Divo (Côte d'Ivoire). Mémoire de D.E.A d'Ecologie tropicale Option Végétale. Université de Cocody Abidjan, Côte d'Ivoire, 65 pp.

N'guessan K., 1995. Contribution à l'étude ethnobotanique en pays Krobou. Thèse de Doctorat de 3è cycle. Faculté des Sciences et Techniques, Université Nationale de Côte d'Ivoire, Abidjan, 583 pp.

Noumi E., 1984. Les plantes à épices, à condiments et à aromates du Cameroun. Thèse de Doctorat $3^{\text {ème }}$ cycle, Université de Yaoundé, Cameroun, 166 pp.

Ouattara D., 1999. Contribution à l'étude Ethnobotanique de la Forêt classée de Dassioko (Fresco, Côte d'ivoire). Mémoire de D.E.A d'Ecologie tropicale (Option : Végétale). Université de Cocody-Abidjan, Côte d'Ivoire, 93 pp.

Osseni B. \& Silue S. J., 1998. Renforcement du rôle des Universités et grandes écoles au sein du Système National de recherche agricole de la Côte d'Ivoire. Mémoire de fin de cycle ingénieur ESA Yamoussoukro, 102 pp.

Tabuna H., 1999. Le marché des produits forestiers non ligneux de l'Afrique centrale en France et Belgique. Produits, acteurs, circuits de distribution et débouchés actuels. Document spécial du CIFOR n¹9. CIFOR, Bogor, Indonésie. In : Bonnehin L. 2000. Domestication paysanne des arbres fruitiers forestiers. Cas de Coula edulis Baill., Olacaceae et Tieghemella heckelii Pierre ex A. Chev., Sapotaceae, autour du Parc National de Taï, Côte d'Ivoire. Tropenbos-Côte d'Ivoire. Série 1, 138 p.

Tra Bi, F. H., 1997. Utilisations des plantes, par l'homme, dans les Forêts Classées du HautSassandra et de Scio, Côte d'Ivoire. Thèse de Doctorat de $3^{e}$ cycle. UFR Biosciences, Université Cocody-Abidjan, Côte d'Ivoire, 215 pp. 
Annexe 1: Liste des espèces inventoriées

\begin{tabular}{|c|c|c|c|}
\hline Noms des espèces & Familles & Noms locaux en Bété & $\begin{array}{c}\text { Forme } \\
\text { biologique }\end{array}$ \\
\hline $\begin{array}{l}\text { Acalypha ciliata Forsk } \\
\text { Aframomum sceptrum (Oliv. \& Hamb.) K. Schum. } \\
\text { Laccosperma secundiflorum (P.Beauv.) O. Kuntze. } \\
\text { Beilschmiedia mannii (Meisn) Benth. \& Hook } \\
\text { Bidens pilosa Linn. }\end{array}$ & $\begin{array}{l}\text { Euphorbiaceae } \\
\text { Zingiberaceae } \\
\text { Arecaceae } \\
\text { Lauraceae } \\
\text { Asteraceae }\end{array}$ & $\begin{array}{l}\text { Mamou } \\
\text { Mênion } \\
\text { Gbolo } \\
\text { Bitéi } \\
\text { Kpakasoisoi }\end{array}$ & $\begin{array}{l}\text { Herbacée } \\
\text { Herbacée } \\
\text { Fausse liane } \\
\text { Arbre } \\
\text { Herbacée }\end{array}$ \\
\hline Blighia sapida Konig & Sapindaceae & Kpagoué & Arbre \\
\hline $\begin{array}{l}\text { Bombax buonopozense P. Beauv. } \\
\text { Buchholzia coriacea Engl. } \\
\text { Calamus deërratus (Linn) Mann. \& Wendl. } \\
\text { Calpocalyx aubrevillei Pellegr. } \\
\text { Calpocalyx brevibracteatus Hams. } \\
\text { Capsicum frutescens Linn. }\end{array}$ & $\begin{array}{l}\text { Bombacaceae } \\
\text { Capparaceae } \\
\text { Arecaceae } \\
\text { Mimosaceae } \\
\text { Mimosaceae } \\
\text { Solanaceae }\end{array}$ & $\begin{array}{l}\text { Wonbou Goda } \\
\text { Lébé } \\
\text { Gbélé } \\
\text { Gnépeu;Gnépo } \\
\text { Youkouta-pia } \\
\text { Déclé; Zi-gnon }\end{array}$ & $\begin{array}{l}\text { Arbre } \\
\text { Arbre } \\
\text { Fausse liane } \\
\text { Arbre } \\
\text { Arbre } \\
\text { Arbuste }\end{array}$ \\
\hline Carpolobia lutea G. Don & Polygalaceae & Kopé & Arbuste \\
\hline $\begin{array}{l}\text { Cayratia gracilis (Guill. \& Pell.) } \\
\text { Ceiba pentandra (Linn.) Gaernt. }\end{array}$ & $\begin{array}{l}\text { Vitaceae } \\
\text { Bombacaceae }\end{array}$ & $\begin{array}{l}\text { Koyoro } \\
\text { Go }\end{array}$ & $\begin{array}{l}\text { Liane } \\
\text { Arbre }\end{array}$ \\
\hline Chrysophyllum perpulchrum Hutch.\&Dalz.. & Sapotaceae & Kpaclé & Arbre \\
\hline $\begin{array}{l}\text { Cissus adenocaulis Stend. (Berhaut) } \\
\text { Cleome ciliata Schum. \& Thonn. } \\
\text { Clerodendrum splendens (G.Don) A. Chev. } \\
\text { Cola millenii K. Schum.. } \\
\text { Cola gigantea var.glabrescens Brenan et Keay } \\
\text { Cola lateritia Schum. var. maclaudii (A. Chev.) } \\
\text { Brenan \& Keay }\end{array}$ & $\begin{array}{l}\text { Vitaceae } \\
\text { Capparidaceae } \\
\text { Verbenaceae } \\
\text { Sterculiaceae } \\
\text { Sterculiaceae } \\
\text { Sterculiaceae }\end{array}$ & $\begin{array}{l}\text { Inconnu } \\
\text { Gnamè } \\
\text { Moutrou-man } \\
\text { Bô ; Bouti } \\
\text { Bô;Bouti } \\
\text { Bô ;Bouti }\end{array}$ & $\begin{array}{l}\text { Liane } \\
\text { Herbacée } \\
\text { Liane } \\
\text { Arbre } \\
\text { Arbre } \\
\text { Arbuste }\end{array}$ \\
\hline Corchorus olitorus Linn. & Tiliaceae & Kprala & Herbacée \\
\hline $\begin{array}{l}\text { Coula edulis Baill. } \\
\text { Cucumeropsis edulis Naud. } \\
\text { Dacryodes klaineana (Pierre) Lam. } \\
\text { Deinbollia pinnata (Poir.) Schum. \& Thonn. } \\
\text { Dioscorea odoratissima Pax } \\
\text { Dioscorea smilacifolia De Wild. } \\
\text { Discorea praehensilis Benth. } \\
\text { Elaeis guineensis Jacq. } \\
\text { Garcinia kola Heckel } \\
\text { Glyphaea brevis (Spreng) Monachino } \\
\text { Grewia mollis Juss. } \\
\text { Ipomea obscura (L.) Ker-caul. var. Obscura } \\
\text { Irvingia gabonensis (O'Rorke)Baill }\end{array}$ & $\begin{array}{l}\text { Olacaceae } \\
\text { Cucurbitaceae } \\
\text { Burseraceae } \\
\text { Sapindaceae } \\
\text { Dioscoreaceae } \\
\text { Dioscoreaceae } \\
\text { Dioscoreaceae } \\
\text { Arecaceae } \\
\text { Clusiaceae } \\
\text { Tiliaceae } \\
\text { Tiliaceae } \\
\text { Menispermaceae } \\
\text { Irvingiaceae }\end{array}$ & $\begin{array}{l}\text { Gnoubê } \\
\text { Gnonsolo } \\
\text { Pêsro; Sênion } \\
\text { Pouna-grahi } \\
\text { Sèmè ; Sèbè } \\
\text { Têtêgbiha } \\
\text { Clima } \\
\text { Goui } \\
\text { Djalouï } \\
\text { Louka-siéné } \\
\text { Vétéi } \\
\text { Soté } \\
\text { Suoko }\end{array}$ & $\begin{array}{l}\text { Arbre } \\
\text { Liane } \\
\text { Arbre } \\
\text { Arbuste } \\
\text { Liane } \\
\text { Liane } \\
\text { Liane } \\
\text { Faux arbre } \\
\text { Arbre } \\
\text { Arbuste } \\
\text { Arbuste } \\
\text { Liane } \\
\text { Arbre }\end{array}$ \\
\hline Isolona campanulata Engl.Diels & Annonaceae & Têbê-kêkêli & Arbuste \\
\hline $\begin{array}{l}\text { Justicia flora (Vahl) Vahl } \\
\text { Landolphia hirsuta (Hua) Pichon } \\
\text { Landolphia owariensis P. Beauv. } \\
\text { Laportea aeatuans (Linn.) Chev. } \\
\text { Momordica cabraei (Cogn.) Jeffrey } \\
\text { Myrianthus arboreus P. Beauv. } \\
\text { Myrianthus libericus Rendle } \\
\text { Myrianthus serratus (Trécul) Benth \& Hook. } \\
\text { Ocimum gratissimum Linn. } \\
\text { Parkia bicolor A. Chev. } \\
\text { Passiflora foetida Linn. } \\
\text { Piper guineense Schum. \& Thonn. } \\
\text { Psychotria psychotrioides (DC.) Roberty } \\
\text { Raphia hookeri Mann. \& Wendl. } \\
\text { Ricinodendron heudelotii (Baill.) Heckel } \\
\text { Salacia oliveriana Loes. var. oliveriana } \\
\text { Sesamum indicum }\end{array}$ & $\begin{array}{l}\text { Canthaceae } \\
\text { Apocynaceae } \\
\text { Apocynaceae } \\
\text { Urtaceae } \\
\text { Cucurbitaceae } \\
\text { Cecropiaceae } \\
\text { Cecropiaceae } \\
\text { Cecropiaceae } \\
\text { Lamiaceae } \\
\text { Mimosaceae } \\
\text { Passifloraceae } \\
\text { Piperaceae } \\
\text { Rubiaceae } \\
\text { Arecaceae } \\
\text { Euphorbiaceae } \\
\text { Hyppocrateaceae } \\
\text { Pedalliaceae }\end{array}$ & $\begin{array}{l}\text { Djédjé-Niko } \\
\text { Lêdé } \\
\text { Zatè, Klouadikpa } \\
\text { Gbaha-gbaha } \\
\text { Gblimion } \\
\text { Wou-ticliti } \\
\text { Bago-ticliti } \\
\text { Wondi-ticliti } \\
\text { Guinguin-nia } \\
\text { Gbligo } \\
\text { Trobi } \\
\text { Gnan, Gnin } \\
\text { Zalakalé } \\
\text { Loulou, Loulè } \\
\text { Kô } \\
\text { Portô } \\
\text { Mahiko }\end{array}$ & $\begin{array}{l}\text { Herbacée } \\
\text { Liane } \\
\text { Liane } \\
\text { Herbacée } \\
\text { Liane } \\
\text { Arbre } \\
\text { Arbre } \\
\text { Arbre } \\
\text { Arbuste } \\
\text { Arbre } \\
\text { Liane } \\
\text { Liane } \\
\text { Arbuste } \\
\text { Faux arbre } \\
\text { Arbre } \\
\text { Liane } \\
\text { Herbe }\end{array}$ \\
\hline Solanum indicum Linn. & Solanaceae & Télékpa-kpa & Arbuste \\
\hline Solanum nigrum Linn. & Solanaceae & Négbo-koua & Herbacée \\
\hline Solanum torvum Sw. & Solanaceae & Gnêzêlé & Arbuste \\
\hline $\begin{array}{l}\text { Sphenocentrum jollyanum Pierre } \\
\text { Spondias mombin Linn. } \\
\text { Sterculia tragacantha Lindl }\end{array}$ & $\begin{array}{l}\text { Menispermaceae } \\
\text { Anacardiaceae } \\
\text { Sterculiaceae }\end{array}$ & $\begin{array}{l}\text { Gnoudi-djapo } \\
\text { Tété } \\
\text { Zêglê-poutouhi }\end{array}$ & $\begin{array}{l}\text { Arbuste } \\
\text { Arbre } \\
\text { Arbre }\end{array}$ \\
\hline $\begin{array}{l}\text { Strombosia pustulata Oliv. var. lucida (Léonard) } \\
\text { Villiers }\end{array}$ & Olacaceae & Klê-kô & Arbre \\
\hline $\begin{array}{l}\text { Telfairia occidentalis Hook. f. } \\
\text { Tetracera alnifolia Wild. }\end{array}$ & $\begin{array}{l}\text { Cucurbitaceae } \\
\text { Dillaniaceae }\end{array}$ & $\begin{array}{l}\text { Djikoun } \\
\text { Dêgni }\end{array}$ & $\begin{array}{l}\text { Liane } \\
\text { Liane }\end{array}$ \\
\hline $\begin{array}{l}\text { Thaumatococcus daniellii (Bennet) Benth. \& Hook. } \\
\text { Tieghemella heckelii Pierre ex A. Chev. }\end{array}$ & $\begin{array}{l}\text { Marantaceae } \\
\text { Sapotaceae }\end{array}$ & $\begin{array}{l}\text { Gbogli } \\
\text { Guézéh }\end{array}$ & $\begin{array}{l}\text { Herbacée } \\
\text { Arbre }\end{array}$ \\
\hline $\begin{array}{l}\text { Tieghemella heckelii Pierre ex A. Chev. } \\
\text { Treculia Africana Desc. subsp. africana var. Africana }\end{array}$ & $\begin{array}{l}\text { Sapotaceae } \\
\text { Moraceae }\end{array}$ & $\begin{array}{l}\text { Guézéhi } \\
\text { Nonhou }\end{array}$ & $\begin{array}{l}\text { Arbre } \\
\text { Arbre }\end{array}$ \\
\hline Trichoscypha arborea (A. Chev.) A. Chev. & Anacardiaceae & Boudrou & Arbre \\
\hline Triplochiton scleroxylon K.Schum & Sterculiaceae & Gligbeu & Arbre \\
\hline Wissadula amplissima (L.) R. E. Fries & Malvaceae & Gboudouzo-zopo & liane \\
\hline Xylopia aethiopica (Dunal) A. Rich. & Annonaceae & Lilo, Liloï & Arbre \\
\hline Zanthoxylum gilletii (De Willd.) Waterman & Rutaceae & Gbessi & Arbre \\
\hline Zanthoxylum rubescens Hook.f. & Rutaceae & Wonnou & Arbre \\
\hline
\end{tabular}

